\title{
Electromagnetic Modeling of the Propagation Characteristics of Satellite Communications Through Composite Precipitation Layers, Part1: Mathematical Formulation
}

\author{
${ }^{*}$ H.M.Al-Rizzo, ${ }^{* *}$ H.T. Al-Hafid, and ${ }^{* * *}$ J.M.Tranquilla
}

\begin{abstract}
${ }^{*}$ College of Information Science and Systems Engineering, University of Arkansas, 2801 South, University Avenue, Little Rock, AR 72204; and Department of Electrical and Electronics Engineering, College of Engineering, Sultan Qaboos University, P.O. Box 33, Al Khod 123, Muscat, Sultanate of Oman; ${ }^{* *}$ Electrical Engineering Department, United Arab Emirates University, Al Ain, United Arab Emirates; ${ }^{* \star *}$ EMR Microwave Technology Corporation, 64 Alison Blvd., Suite 105, Fredericton, NB, Canada, E3C 1N2.
\end{abstract}
نمنجة انتشار الموجلت الكهرومغنطيسية لمظومات الأقمار الصناعية خللاطقات المطر المترلكمة : النموذج الرياضئي

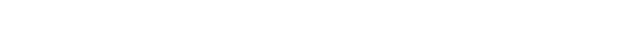

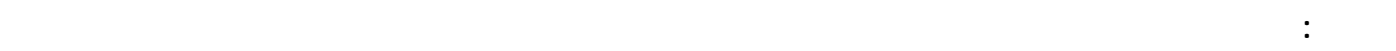

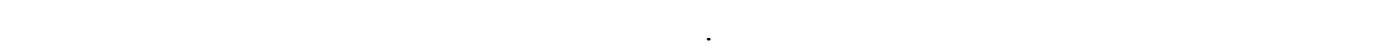

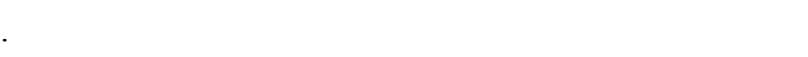

\begin{abstract}
A systematic and general formulation of a Propagation Simulation Program (PSP) is developed for the coherent field of microwave and millimeter wave carrier signals traversing intermediate layered precipitation media, taking into account the random behavior of particle size, orientation, shape and concentration distributions. Based on a rigorous solution of the volumetric multiple-scattering integral equations, the formalism offers the capability of treating the potential transmission impairments on satelliteearth links and radar remote sensing generated by composite atmospheric layers of precipitation in conjunction with the finite polarization isolation of dual-polarized transmitting and receiving antennas. A multi-layered formulation is employed which encompasses an ensemble of discrete particles comprising an arbitrary mixture of ice crystals, melting snow and raindrops that may exist simultaneously along satellite-earth communication paths.
\end{abstract}

KEYWORDS: Microwave, Multiple-Scattering, Remote Sensing.

$\mathbf{R}$

ecent demand for additional communication capacity, combined with the requirement of

alleviating the congested electromagnetic (EM) spectrum, has led system planners to utilize increasingly higher carrier frequencies. Many attractive features for earth-space communications, radar, remote sensing and imaging applications such as improved resolution and wide absolute bandwidths essential for civilian and military high data rate transmission needs are feasible in the millimeter wave (MMW) regime. In this paper the analytical formulation of a PSP, based on a rigorous solution of the volumetric multiple-scattering integral equations (Ishimaru, 1977), is presented to evaluate the impairments caused by precipitation along arbitrary slant earth-space paths 
utilizing the Generalized Multipole Technique (GMT) developed by Al-Rizzo and Tranquilla (1997). A concise matrix approach is employed for the treatment of the ensemble-averaged, coherent field of MMW signals traversing a random collection of hydrometeors including ice crystals, melting snow and rainfall.

The purpose of the work reported in this paper is twofold. First, to integrate the simulation through the whole spectrum of precipitation using a multi-layered formulation which includes inhomogeneous mixture of ice at top of rain clouds and the transition from melting snow to rain below the melting layer. The second objective, and perhaps the foremost, is to extend the frequency range and improve the accuracy of previously published results, within the limitations of available meteorological data and physical parameters concerning the various forms of hydrometeors in the lower part of the atmosphere.

\section{General Medium Modelling}

An arbitrarily polarized time-harmonic incident plane EM wave, propagating in the positive $z$ direction, is assumed. The polarization state is expressed in terms of the angle pair, $(\gamma, \delta)$, where $\gamma=$ $\tan ^{-1}\left(E_{2} / E_{1}\right), E_{1}$ and $E_{2}$ are the maximum projections of the instantaneous electric field vector along the $x$ and $y$ axes, respectively, and $\delta$ is the time-phase angle by which $E_{y}$ leads $E_{x}$ (Kraus and Carver, 1973).

A Cartesian coordinate system is employed, the $z$-axis defines the incident wave direction, pointing from the satellite antenna toward the receiving antenna at the earth-terminal location. The $x$ and $y$ axes coincide with the reference horizontal and vertical directions, respectively, forming a right-handed triplet where the $x$-axis is parallel to the local horizontal at the receiving end.

Complex normalized column vectors; $\boldsymbol{V}_{t}$ of impressed and $\boldsymbol{V}_{r}$ of received phasor voltages are defined such that $P_{t i}=V_{t i} . V_{t i}^{*}$ is the input power to the transmitting antenna and $P_{r i}=V_{r i} . V_{r i}^{*} i=$ 1,2 is the power delivered to a matched load by the receiving antenna. The finite polarization isolation, inherently present in the transmitting and receiving antennas, is accounted for via two matrices; $\left[\boldsymbol{A}_{t}\right]$ and $\left[\boldsymbol{A}_{r}\right]$ which relate the incident $\boldsymbol{E}_{i}$ and received $\boldsymbol{E}_{r}$ electric field vectors to $\boldsymbol{V}_{t}$ and $\boldsymbol{V}_{r}$ such that $\boldsymbol{E}_{\boldsymbol{i}}=\left[\boldsymbol{A}_{t}\right] \boldsymbol{V}_{t}$ and $\boldsymbol{V}_{r}=\left[\boldsymbol{A}_{r}\right] \boldsymbol{E}_{r}$ where (Bostian et al 1982)

$$
\begin{aligned}
& {\left[\begin{array}{c}
E_{i}^{x} \\
E_{i}^{y}
\end{array}\right]=\left[\begin{array}{cc}
\cos \gamma_{t 1} & \cos \gamma_{t 2} \\
\sin \gamma_{t 1} e^{j \delta_{t 1}} & \sin \gamma_{t 2} e^{j \delta_{t 2}}
\end{array}\right]\left[\begin{array}{c}
V_{t 1} \\
V_{t 2}
\end{array}\right]} \\
& {\left[\begin{array}{l}
V_{r 1} \\
V_{r 2}
\end{array}\right]=\left[\begin{array}{ll}
\cos \gamma_{r 1} & \sin \gamma_{r 1} e^{-j \delta_{r 1}} \\
\cos \gamma_{r 2} & \sin \gamma_{r 2} e^{-j \delta_{r 2}}
\end{array}\right]\left[\begin{array}{c}
E_{r}^{x} \\
E_{r}^{y}
\end{array}\right]}
\end{aligned}
$$

The subscript $t(r)$ refers to the transmitting (receiving) antenna, respectively and the two (nominally) orthogonal polarizations are denoted by the additional subscript 1 (2).

The coherent electric field vector exiting a discrete, random anisotropic and lossy dielectric medium is related to the corresponding incident field using the eigenvalues and eigenvectors of the ensembleaveraged, complex propagation constant matrix $\left[<\boldsymbol{k}_{l}>\right]$, the exponential form of which can be expressed as (Tsoloakis and Stutzman, 1982)

$$
\left[\begin{array}{l}
E_{r}^{x} \\
E_{r}^{y}
\end{array}\right]=e^{-j k_{0} L}[\boldsymbol{Q}]\left[\begin{array}{cc}
e^{-j \lambda_{1}} & 0 \\
0 & e^{-j \lambda_{2}}
\end{array}\right]\left[\boldsymbol{Q}^{-1}\right]\left[\begin{array}{l}
E_{i}^{x} \\
E_{i}^{y}
\end{array}\right]
$$

where $L$ is the path length along the propagation direction and $k_{o}$ is the free-space wave number. The term $e^{-j k_{o} L}$ characterizes the pure phase-shift properties of the scatterer-free medium and $[Q]$ is the matrix of non-trivial eigenvector solutions of $\left[<\boldsymbol{k}_{l}>\right]$ which are found from 


$$
\begin{gathered}
\lambda_{1,2}=\frac{\left\langle k_{11}\right\rangle+\left\langle k_{22}\right\rangle \pm \sqrt{\left(\left\langle k_{11}\right\rangle-\left\langle k_{22}\right\rangle\right)^{2}+\left(2\left\langle k_{12}\right\rangle\right)^{2}}}{2} \\
{[\boldsymbol{Q}]=\left[\begin{array}{cc}
\left\langle k_{22}\right\rangle-\lambda_{1} & \left\langle k_{22}\right\rangle-\lambda_{2} \\
\left\langle-k_{12}\right\rangle & \left\langle-k_{12}\right\rangle
\end{array}\right]}
\end{gathered}
$$

When the matrix of eigenvectors and its inverse are substituted into (2), then (2) can be transformed into the following concise form

$$
\boldsymbol{E}_{r}(L)=e^{-j k_{o} L}[<\boldsymbol{T}>] \boldsymbol{E}_{i}(L)
$$

The elements of the transmission matrix $[<\boldsymbol{T}>$, which describes the amplitude, phase and polarization of the coherent field exiting the medium, can be written as

$$
\begin{gathered}
\left\langle T_{11}\right\rangle=\frac{\left(\left\langle k_{22}\right\rangle-\lambda_{2}\right) e^{-j \lambda_{2}}-\left(\left\langle k_{22}\right\rangle-\lambda_{1}\right) e^{-j \lambda_{1}}}{\lambda_{1}-\lambda_{2}} \\
\left\langle T_{12}\right\rangle=\left\langle T_{21}\right\rangle=-\left\langle k_{12}\right\rangle \frac{e^{-j \lambda_{2}}-e^{-j \lambda_{1}}}{\lambda_{1}-\lambda_{2}} \\
\left\langle T_{22}\right\rangle=\frac{\left(\left\langle k_{22}\right\rangle-\lambda_{2}\right) e^{-j \lambda_{1}}-\left(\left\langle k_{22}\right\rangle-\lambda_{1}\right) e^{-j \lambda_{2}}}{\lambda_{1}-\lambda_{2}}
\end{gathered}
$$

The transfer-matrix equation describing the input-output relationship of the complete orthogonalpolarized, frequency re-use system can then be found by combining (1) and (5) as

$$
\boldsymbol{V}_{r}=e^{-j k_{O} L}\left[\boldsymbol{A}_{\boldsymbol{r}}\right][<\boldsymbol{T}>]\left[\boldsymbol{A}_{\boldsymbol{t}}\right] \boldsymbol{V}_{t}
$$

\section{Theoretical Development For The Rainfall Medium}

A PSP must include empirical or statistical averaging to account for the temporal and spatial randomness of the rain characteristics. Falling raindrops are nonspherical in shape and are influenced by aerodynamic forces. As the size of a raindrop increases, a concave depression develops. Pruppacher and Pitter (1971),(P-P), provided 13 realistic raindrop shapes with spherical equivolume radii from 0.25 to $3.25 \mathrm{~mm}$. With the exception of the limited cases presented by Oguchi (1977), Fang and Lee (1978) and Li et al. (1995), previous models considered only spherical and/or oblate spheroidal raindrops. The GMT is utilized in this paper to compute the forward scattering functions of an oblate and/or P-P raindrop, the symmetry axis of which coincides with the $z$-axis as shown in Figure 1. A plane EM wave, incident in the $\boldsymbol{k}_{i}$ direction and inclined at an angle $\beta$ with respect to the horizontal plane, is assumed. The incident electric field vector is given by

$$
\boldsymbol{E}_{i}=\boldsymbol{i} e^{-j \boldsymbol{k}_{i} \cdot \boldsymbol{r}}
$$

where the unit vector $\boldsymbol{i}$ describes the polarization state of the incident field.

The propagation direction and the raindrop's axis of rotational symmetry define the incident plane. The incident electric field vector is decomposed into two mutually orthogonal components, parallel $\boldsymbol{E}_{i}^{\amalg}$ and perpendicular $\boldsymbol{E}_{\boldsymbol{i}}^{\perp}$ to the plane of incidence, respectively, as

$$
\boldsymbol{E}_{i}=\boldsymbol{E}_{i}^{\amalg} \boldsymbol{i}_{i}^{\amalg}+\boldsymbol{E}_{i}^{\perp} \boldsymbol{i}_{i}^{\perp}
$$


In the far-field region, the forward-scattered wave has the same polarization as the incident field and the scattering process can be modeled by the following diagonal matrix equation

$$
\left[\begin{array}{c}
E_{s}^{\perp} \\
E_{s}^{\amalg}
\end{array}\right]=\frac{e^{-j k_{0} r}}{r}\left[\begin{array}{cc}
f_{\perp, \perp}\left(a_{0}, \beta, \varepsilon_{w}\right) & 0 \\
0 & f_{\amalg, \amalg}\left(a_{0}, \beta, \varepsilon_{w}\right)
\end{array}\right]\left[\begin{array}{c}
E_{i}^{\perp} \\
E_{i}^{\amalg}
\end{array}\right]
$$

where $f_{/ /, / /\left(a_{0}, \beta, \varepsilon_{w}\right)}$ and $f_{\perp, \perp}\left(a_{0}, \beta, \varepsilon_{w}\right)$ are the forward scattering amplitude functions for the two principal incident polarizations and $\varepsilon_{w}$ is the complex relative dielectric constant of water.

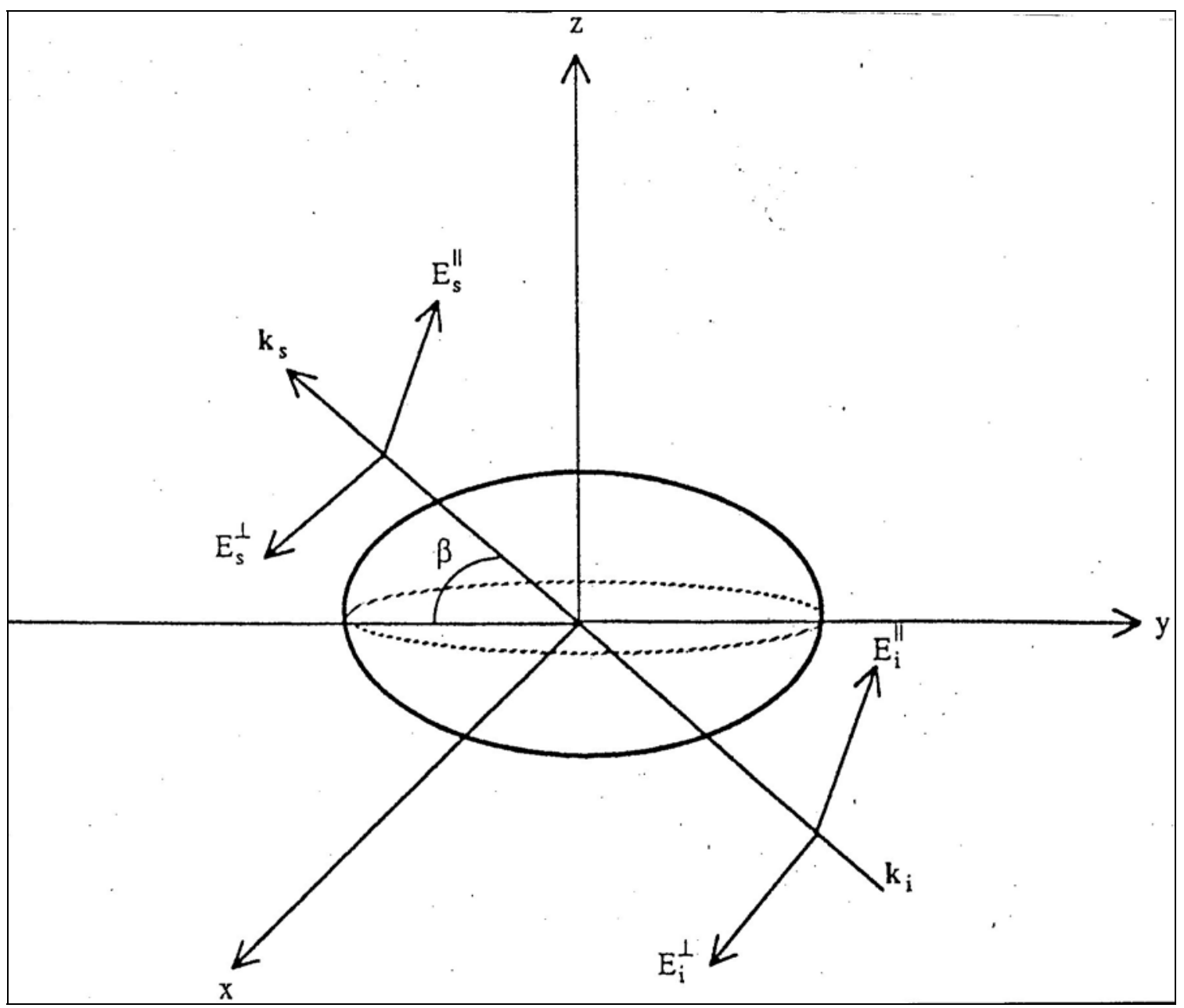

Figure 1. Geometry for the scattering from an axisymmetric raindrop.

At a given elevation angle, $\beta$ and drop temperature, $f_{/ /, / /\left(a_{0}, \beta, \varepsilon_{w}\right)}$ and $f_{\perp, \perp}\left(a_{0}, \beta, \varepsilon_{w}\right)$ are computed for 13 drops with spherical equivolume radii from $0.25 \mathrm{~mm}$ to $3.5 \mathrm{~mm}$ within a convergence criterion of $10^{-4}$ for $a_{0} \leq 2 \mathrm{~mm}$ and $10^{-3}$ for $a_{0} \geq 2 \mathrm{~mm}$ for the two principal polarizations. The integrals of the complex-valued scattering functions over the appropriate raindrop size distribution have been numerically evaluated using a 14-point composite Simpson's rule.

The orientation geometry of an oblate and/or P-P falling raindrop is defined in the $x-y$ plane in terms of the apparent canting angle between the projection of the symmetry axis and the reference polarization directions (Oguchi, 1977). For an assemblage of identically oriented raindrops, a diagonal transmission matrix is defined, the diagonal elements describe the coherent attenuation and phase shift encountered by the principal polarizations. If the canting angle is denoted by $\theta$, a matrix transformation is performed to bring the reference polarizations into the principal axes of the raindrop via the rotation matrix 


\section{MODELING THE PROPAGATION OF SATELLITE COMMUNICATIONS}

$$
\boldsymbol{R}_{\theta}=\left[\begin{array}{cc}
\cos \theta & \sin \theta \\
-\sin \theta & \cos \theta
\end{array}\right]
$$

The components of the forward-scattered electric field vector are next rotated back into the reference $x$ and $y$ directions by applying the inverse rotation $R_{\theta}^{-1}$.

The ensemble-averaged, complex propagation constant matrix, $\left[\left\langle\boldsymbol{k}_{1}\right\rangle\right]$ which characterizes the coherent forward scattering effects due to a population of raindrops with a prescribed size, canting angle and shape distributions can then be written as

$$
\left[\left\langle\boldsymbol{k}_{1}\right\rangle\right]=\frac{2 \pi}{k_{0}} \iiint[\boldsymbol{f}] N\left(a_{0}, \theta, s, z\right) d a_{0} d \theta d s
$$

where $N\left(a_{0}, \theta, s, z\right)$ is the number of raindrops of a given shape, $s$, within a specified size, $a_{0}$, and orientation, $\theta$, intervals per unit volume of space which is a function of the spatial variable, $z$ along the propagation direction.

The forward-scattering matrix obtained from (10) after applying the rotation matrix of (11) and its inverse, when averaged over the statistical parameters of shape and canting angle distributions, can be written as

$$
\begin{gathered}
\left\langle f_{11}\right\rangle=P_{s} f_{s}\left(a_{0}\right)+\left(1-P_{s}\right)\left\{f_{\perp, \perp}\left\langle\cos ^{2} \theta\right\rangle+f_{\mathrm{L}, \amalg}\left\langle\sin ^{2} \theta\right\rangle\right\} \\
\left\langle f_{12}\right\rangle=\left\langle f_{21}\right\rangle=\left(1-P_{s}\right)\left\{f_{\perp, \perp}-f_{\amalg, \amalg}\right\} \frac{\langle\sin 2 \theta\rangle}{2} \\
\left\langle f_{22}\right\rangle=P_{s} f_{s}\left(a_{0}\right)+\left(1-P_{s}\right)\left\{f_{\perp, \perp}\left\langle\sin ^{2} \theta\right\rangle+f_{\mathrm{U}, \amalg}\left\langle\cos ^{2} \theta\right\rangle\right\}
\end{gathered}
$$

where $f_{S}$ is the forward scattering amplitude of a spherical water drop. The common assumption of statistically independent shape and canting angle distributions has been adopted in (13). A discrete bimodal shape distribution is assumed, $P_{S}$ being the effective fraction of spherical and the remaining $\left(1-P_{S}\right)$ are oblate and/or P-P drops.

For linearly polarized geo-stationary satellite transmission, the horizontal and vertical orientations at the satellite are referenced with respect to the subsatellite point as either in or perpendicular to the plane of the equator, respectively. For earth stations not located on the meridian, the electric field vector appears tilted from the local vertical or horizontal by an angle $\tau$ where (Allnutt, 1989)

$$
\tau=90^{\circ}-\tan ^{-1}\left[\frac{\tan \xi}{\sin \eta}\right]
$$

and $\xi$ and $\eta$ are the longitude and latitude of the earth station, respectively, defined with respect to the subsatellite point. If the receiving antenna is aligned to the satellite transmission, then equation (7) must be modified to

$$
\boldsymbol{V}_{r}=e^{-j k} 0^{L}\left[\boldsymbol{A}_{r}\right]\left[\boldsymbol{R}_{\tau}\right]^{-1}[\langle\boldsymbol{T}\rangle]\left[\boldsymbol{R}_{\tau}\right]\left[\boldsymbol{A}_{t}\right] \boldsymbol{V}_{t}
$$

where the rotation matrix

$$
\boldsymbol{R}_{\tau}=\left[\begin{array}{cc}
\cos \tau & \sin \tau \\
-\sin \tau & \cos \tau
\end{array}\right]
$$


transforms the satellite's vertical and horizontal to the local vertical and horizontal at the earth station.

\section{Theoretical Development For The Melting - Layer Medium}

The melting layer is a prominent feature of widespread rain associated with stratiform precipitation. It contains a mixture of gradually melting snowflake aggregates, which eventually form raindrops, attaining the terminal fall speed of water upon complete melting at the bottom of the layer (Ekpenyong and Srivastava, 1970). The establishment of a simulation model for the melting layer is imperative to accurately predict the total attenuation and phase shift for lowelevation satellites where a portion of the radio path may be contained within the layer boundary particularly in temperate maritime, northerly latitude and tropical climates.

The meteorological model of the melting process developed by Ekpenyong and Srivastava (1970) is adopted in this paper with a spectrum of polydispersed snowflake sizes, densities and fall speeds. Due to the lack of definitive information on the actual shape and orientation of partially melted snowflakes, the present model assumes a two-layered spherical scatterer shape.

Initially, a dry snowflake of radius $r_{s}$ and density $\rho_{s}$ originating at the top of the melting layer is considered. A two-layered, concentric spherical snowflake of radius $r_{0}$ with the melted water forming a uniform shell around a snow core of radius $r_{i}$ is assumed.

If $y$ denotes the fraction of total mass melted, then

$$
y=\frac{r_{0}^{3}-r_{i}^{3}}{r_{w}^{3}}
$$

where $r_{w}$ is the radius of the resulting water drop at the bottom of the layer.

The particle number density and the terminal fall speed, denoted respectively by $N_{0}$ and $v$, depend on $r_{0}$ and the height of fall $\left(z-z_{0}\right)$ below freezing level where the $0^{0} \mathrm{C}$ isotherm starts at $z$ $=z_{0}, y=0$ at the top of the layer. The precipitation intensity is considered constant throughout the melting region with aggregation and breakup ignored such that a one-to-one correspondence exists between raindrops at the bottom and dry snowflakes at the top. If $N\left(r_{w}\right) d r_{w}$ denotes the raindrop size distribution, the corresponding distribution of the melting snow particles at any height within the melting zone can be estimated from (Dissanayake and McEwan, 1978)

$$
N_{0}\left(r_{0}, z-z_{0}\right) d r_{0}=\frac{v_{w}\left(r_{w}\right)}{v_{0}\left(r_{0}, z-z_{0}\right)} N_{w}\left(r_{w}\right) d r_{w}
$$

Consideration of mass conservation in a certain air volume implies

$$
\rho_{s} r_{s}^{3}=\rho_{s} r_{i}^{3}+\rho_{w}\left(r_{0}^{3}-r_{i}^{3}\right)=\rho_{w} r_{w}^{3}
$$

If the density and kinematic viscosity of air and mass of a snowflake are assumed constants during the melting process, it can be shown that the product $v r$ remains constant that is

$$
v_{s} r_{s}=v_{0} r_{0}=v_{w} r_{w}
$$

From (19) and (20), the terminal fall speed, $v_{s}$ of a dry snowflake can be obtained as

$$
v_{s}=v_{w}\left(\frac{\rho_{s}}{\rho_{w}}\right)^{1 / 3}
$$

At any stage of melting, the fall speed, $v_{0}$, can be obtained from (17), (20) and (21) as 


\section{MODELING THE PROPAGATION OF SATELLITE COMMUNICATIONS}

$$
v_{0}=\frac{v_{s}}{\sqrt[3]{1-\left[1-\left(\frac{v_{s}}{v_{w}}\right)^{3}\right]}}
$$

For a dry snowflake, $\rho_{s}$ approaches a constant value of $0.008 \mathrm{~g} / \mathrm{cm}^{3}$ for $r_{0}>0.75 \mathrm{~cm}$. For smaller radii, $\rho_{s}$ may be taken as $0.3 \mathrm{~g} / \mathrm{cm}^{3}$. In between these two limiting values, the density can be obtained from (Ekpenyong and Srivastava, 1970)

$$
\rho_{s}=\frac{0.005}{r_{s}^{2}}
$$

where $r_{S}$ is in $\mathrm{cm}, \rho_{s}$ is in $\mathrm{g} / \mathrm{cm}^{3}$ and

$$
r_{s}=r_{w}\left(\frac{\rho_{w}}{\rho_{s}}\right)^{1 / 3}
$$

The dielectric constant of the snow core, $\varepsilon_{s}$ is evaluated from the following expression (Dissanayake and McEwan, 1978)

$$
\frac{\varepsilon_{s}-1}{\varepsilon_{s}+2}=\frac{\rho_{s}}{\rho_{i}} \frac{\varepsilon_{i}-1}{\varepsilon_{i}+2}
$$

where a snowflake is treated as a two-component mixture of ice and air, $\varepsilon_{i}, \rho_{i}$ are the complex dielectric constant and density of ice, respectively.

Once the mass fraction of a melted snowflake has been computed, the core and outside radii, terminal fall speed, dielectric constant of the core and particle-size distributions corresponding to a particular size of an eventually melted drop can be evaluated at any prescribed height within the melting layer.

\section{Conclusions}

A theoretical formulation is introduced for the coherent field of a monochromatic EM signal traversing a discrete medium of composite precipitation layers. The model accounts for the random behavior of particle size, orientation, shape and intensity distributions.

Although the degrading effects due to a single tropospheric layer of precipitation, which may hinder reliable space communications, were investigated extensively over the past three decades, the contributions at frequencies in the upper MMW band are still scarce. Furthermore, little has been done to integrate the effects throughout the whole spectrum of precipitation in the lower part of the atmosphere.

\section{References}

ALLNUTT, J.E. 1989. Satellite-to-Ground Radio Wave Propagation, London, U.K.: Peter Peregrinus Ltd.

AL-RIZZO, H.M. and TRANQUILLA, J.M. 1997. Application of the generalized multipole technique (GMT) to highfrequency electromagnetic scattering from perfectly conducting and dielectric bodies of revolution, J. Comput. Phys., 136: $1-18$.

BOSTIAN, C.W, STUTZMAN, W.L. and GRAINES, J.M. 1982. A review of depolarization modeling for earth-space radio paths at frequencies above $10 \mathrm{GHz}$, Radio Sci., 17: 1231-1241.

DISSANAYAKE, A.W. and MCEWAN, N.J. 1978. Radar and attenuation properties of rain and bright band, IEE Conf. Publ. 169-2, 125-129.

EKPENYONG, B.E. and SRIVASTAVA, R.C. 1970. Radar characteristics of the melting layer- A theoretical study, Tech. Rep. No. 16, Dept. of the Geophys. Sci., The University of Chicago and Illinois Inst. of Techn., Dept. of Elect. Eng., Chicago, Illinois.

FANG, D.J. and LEE, F.J. 1978. Tabulation of raindrop induced forward and backward scattering amplitudes, Comsat. Tech. Rev., 8: 455-486. 


\section{AL-RIZZO, AL-HAFID AND TRANQUILLA}

ISHIMARU, A. 1977. Theory and application of wave propagation and scattering in random media, Proc. IEEE, 65: 10301061.

KRAUS, J.D. and CARVER, K.R. 1973. Electromagnetics, New York: McGraw-Hill.

LI LE-WEI, PANG-SHYAN KOOI, MOOK-SENG LEONG, TAT-SOON YEO, and MIN-ZHAN GAO, 1995. Microwave attenuation by realistically distorted raindrops: Part I- Theory, IEEE Trans. Antennas Propagat., AP-43: 811-821.

OGUCHI, T. 1977. Scattering properties of Pruppacher-and-Pitter form raindrops and cross polarization due to rain : calculations at 11, 13, 19.3 and $34.8 \mathrm{GHz}$, Radio Sci., 12: 41-51.

PRUPPACHER, R. and PITTER, R.L. 1971. A semi-empirical determination of the shape of cloud and rain drops, J. Atmos. Sci., 28: 86-94.

TSOLOAKIS, A. and STUTZMAN, W.L. 1982. Multiple scattering of electromagnetic waves by rain, Radio Sci.,12: 14951502.

Received 1 December 1999

Accepted 19 June 2000 